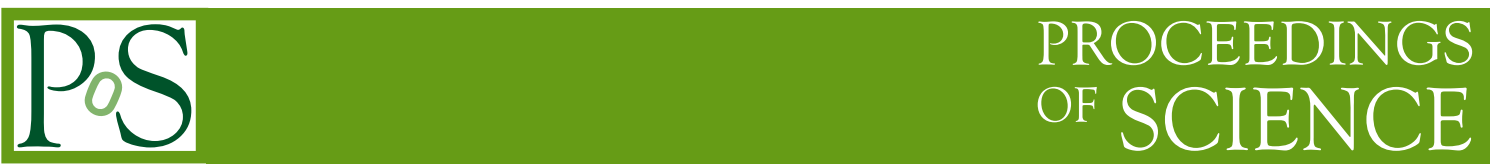

\title{
Highlights from the Telescope Array
}

\author{
J.N. Matthews ${ }^{a *}$, for the Telescope Array Collaboration ${ }^{c}$ \\ ${ }^{a}$ University of Utah, Department of Physics \& Astronomy and High Energy Astrophysics \\ Institute, Salt Lake City, Utah, USA \\ E-mail: jnmephysics.utah.edu
}

Full author list: http://www.telescopearray.org/index.php/research/collaborators

The Telescope Array Project studies the properties of ultra high energy cosmic rays by measuring the extensive air showers which they induce when interacting in the Earth's atmosphere. We do this using a variety of techniques including using an array of scintillator detectors to sample the footprint of the air shower when it reaches the Earth's surface and telescopes to measure the fluorescence and Čerenkov light of the air shower. From this we determine the energy spectrum and chemical composition of the primary particles. We also search for sources of cosmic rays and anisotropy. One of the interesting things which we have found is evidence of a possible source of ultra high energy cosmic rays in the northern sky. The experiment and its recent measurements are discussed.

\footnotetext{
* Speaker.
} 


\section{Introduction}

The Telescope Array collaboration was forged by members of the High Resolution Fly's Eye (HiRes) and Akeno Giant Air Shower Array (AGASA) collaborations. Its purpose is to study ultra high energy cosmic rays, their spectrum, composition, and anisotropy/sources. Additional goals included understanding the differences in the observations of HiRes and AGASA as well as the transition from galactic to extra-galactic sources. The collaboration has, over time, grown to include members from the US, Japan, Russia, South Korea, and Belgium.

The Telescope Array is located in central Utah in the US at about $39.30^{\circ}$ north latitude and $112.91^{\circ}$ west longitude. The location was selected for its dark skies and dry atmosphere to aid in the observation of fluorescence light from the cosmic ray induced extensive air showers. In addition, the altitude of the site is near shower maximum for a vertical shower initiated by a $10^{19} \mathrm{eV}$ cosmic ray. These attributes combine to make a great site for ultra high energy cosmic ray observations.

The high energy component of the experiment consists of 38 telescopes, (9728 PMTs) at three telescope stations overlooking an array of 507 scintillator surface detectors (SD). The SD array has an area greater than $700 \mathrm{~km}^{2}$. The main body of the Telescope Array was complete and operational as of 2008. The layout of the TA experiment is shown in Figure 1.

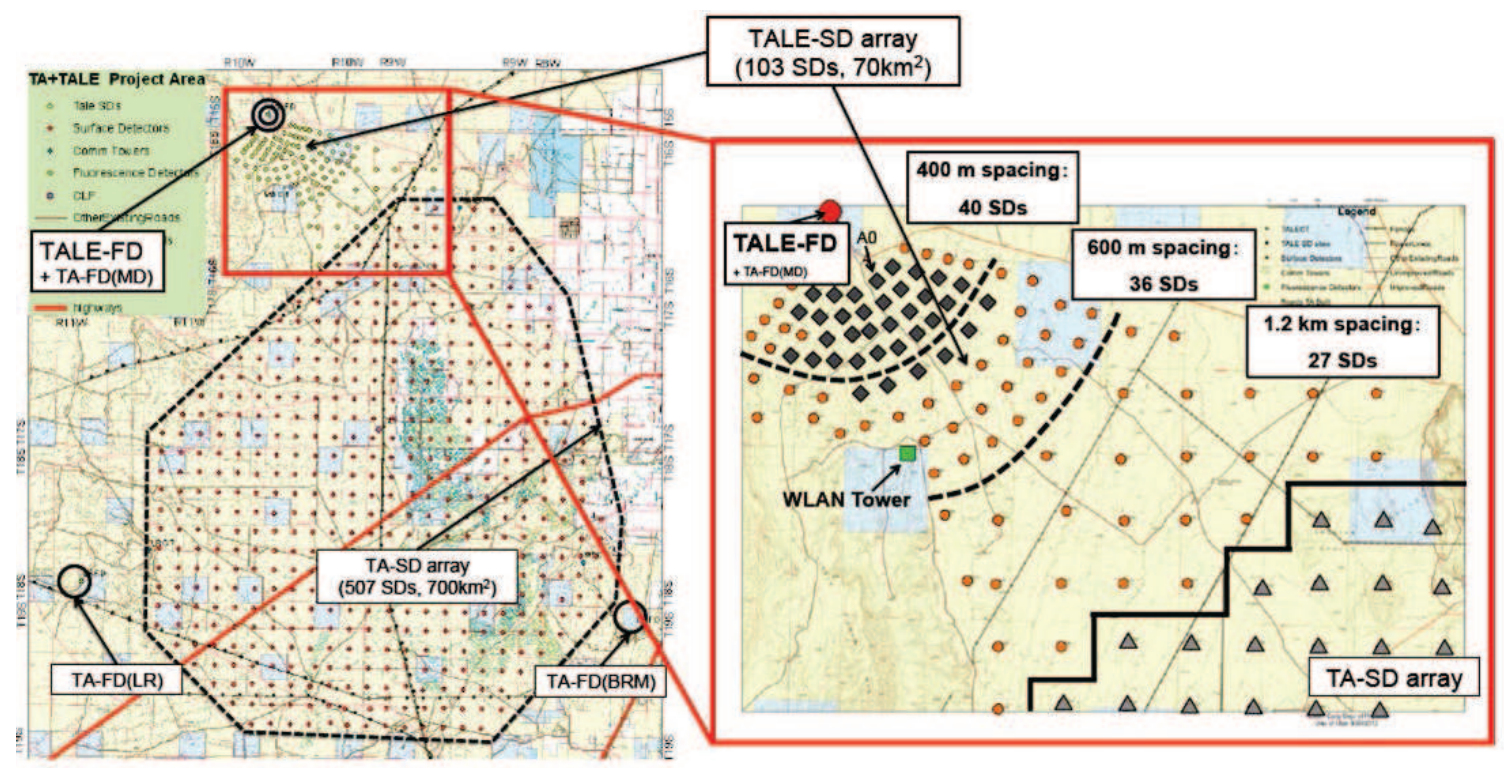

Figure 1: Layout of the Telescope Array detector site. The main array is shown on the left with a surface detector (SD) array of 507 scintillation counters. Three fluorescence detector (FD) stations are on the perimeter (MD: Middle Drum, BRM: Black Rock mesa, LR: Long Ridge) looking inward over the SD. These telescopes view $3^{\circ}-31^{\circ}$ in elevation and $\sim 112^{\circ}$ in azimuth. The expanded inset on the right shows the layout of the TA Low-energy Extension (TALE). The TALE addition to Telescope Array consists of 10 new high-elevation $\left(31^{\circ}-59^{\circ}\right)$ fluorescence telescopes located at the MD site, and an in-fill SD array of 103 counters arranged with spacings that grows with distance from the TALE FD, in order to provide optimal coverage at energies below $10^{18} \mathrm{eV}$.

We use the atmosphere as a part of our calorimeter and observe the energy deposited in the atmosphere by the primary cosmic ray and the extensive air shower it initiates. Telescopes view 
the longitudinal development of the air shower as it traverses the atmosphere. The three telescope stations are located on the perimeter of the SD array (on a $\sim 30 \mathrm{~km}$ triangle) and view the sky over it. The northern telescope station has 14 telescopes, each with a $5.2 \mathrm{~m}^{2}$ mirror. The site re-utilizes telescopes from the High Resolution Fly's Eye, HiRes-I site. The southern two stations are each instrumented with 12 telescopes, with $6.8 \mathrm{~m}^{2}$ mirrors. The new telescopes at these sites use FADC electronics, while the HiRes-I telescopes utilize older sample-and-hold electronics.

The cameras in the telescopes are composed of 256 PMT pixels, each viewing $\sim 1^{\circ}$ of sky. The PMTs are hexagonal and are arranged in a 16x16 hexagonal close pack (honey-comb) array. Each site views from $3-31^{\circ}$ in elevation and has full azimuthal view over the SD array.

The telescopes are looking for an amount of light similar to a 50-100W UV light bulb, but 10-40 km away and moving at the speed of light. Due to the extreme sensitivity of the telescopes and cameras, the telescopes are only operated on clear, moonless nights. In addition, we do not collect data when the weather is poor, such as overcast, rain, snow, or lightning. This results in a duty cycle of about $10 \%$.

The scintillator surface detector (SD) array, samples the density of secondary air shower particles when they reach the Earth's surface. The 507 scintillator surface detectors are each instrumented with two layers of half-inch $(1.25 \mathrm{~cm})$ thick scintillator extruded with grooves in it. Wavelength shifting optical fibers are placed into the grooves. They gather the light generated when particles pass through the detector, shift the wavelength, and guide it to two PMTs, one for each of the optically isolated layers. The two layers sit one on top of the other and each layer samples $3 \mathrm{~m}^{2}$ in area.

The Telescope Array scintillator surface detectors are deployed on a $1.2 \mathrm{~km}$ square grid. They are powered by solar panels and batteries for $24 / 7$ continuous operations. The detectors are selfcalibrated using the muon background and are read out by $2.4 \mathrm{GHz}$ radio. Three radio towers divide the 507 scintillator detectors in the array among them. Each second, the towers poll all of the detectors in their portion of the array for signals larger than three minimum ionizing particles (MIPs), then a computer at the tower scans this data for patterns indicative of a shower footprint. If signs of a footprint are found, then the tower requests that all data greater than $1 / 3$ of a MIP from all of the surface detectors during the relevant time period be sent back and recorded. The towers also communicate with each other to avoid having gaps in sensitivity between the sub-arrays of detectors.

The three telescope stations operate separately and independently from each other and from the SD array. The data from each station can be analyzed separately or in conjunction with the other parts. Data from a single telescope station is known as "mono" data, that from multiple telescope stations is known as "stereo". Data that includes information from a telescope station in addition to that from the surface detector (SD) array is "hybrid" data. Having more information is always better since it constrains the data and reduces the uncertainties. However, one does not always have that luxury - for example the SD data set is the largest since it operates 24/7 (nearly $100 \%$ duty cycle), while the telescopes have a duty cycle of about $10 \%$ due to sun light, moon light, and weather conditions. Hence the SD only data is used where one can tolerate the slightly larger uncertainties such as in the spectrum and anisotropy measurements. Meanwhile, hybrid and stereo data have their own places; these are required to reduce uncertainties enough to make composition measurements. 


\section{Energy Spectrum}

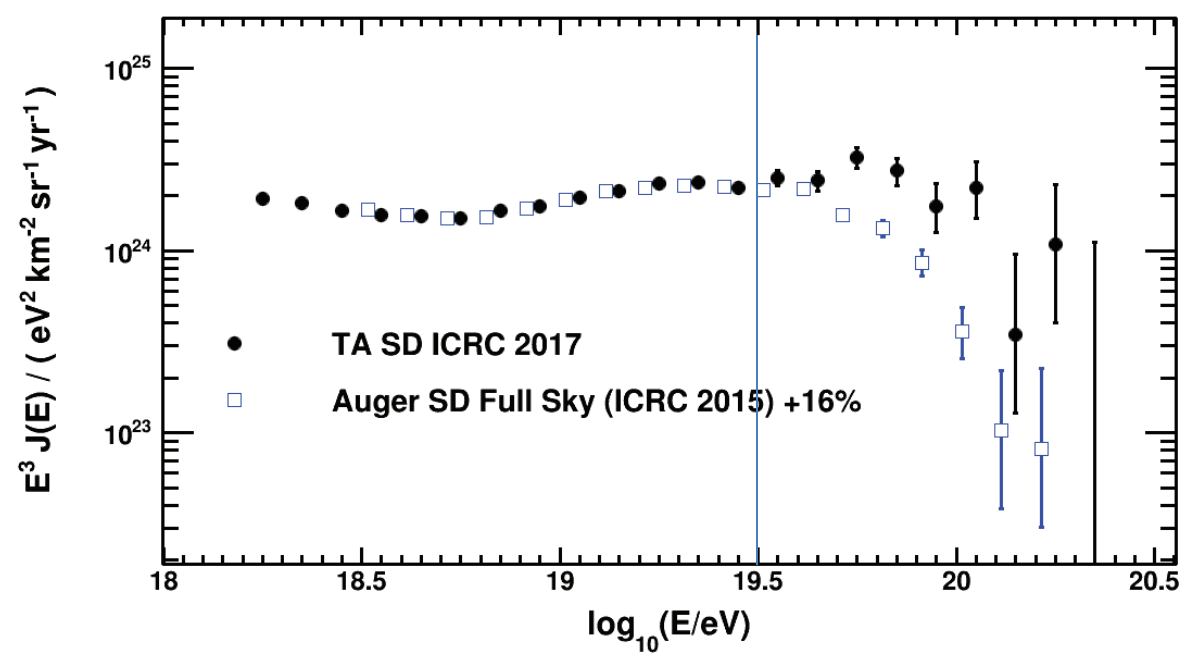

Figure 2: The combined spectrum from the Telescope Array Collaboration, including the first 9 years data from the surface detector spectrum. There is a dip or ankle in the spectrum at $10^{18.67 \pm 0.02} \mathrm{eV}$ and the GZK break occurs As predicted at $10^{19.81 \pm 0.04} \mathrm{eV}$. Integrating an unbroken line from the break to $10^{21} \mathrm{eV}$, one would expect to find $\sim 80$ events, while 22 events are actually observed in this range. The significance of the cut-off is $\sim 7 \sigma$.

The scintillator array has the greatest number of events. Telescope Array previously published an energy spectrum from the first four years of SD operation [1]. The energy spectrum of ultra high energy cosmic rays is now updated to include data from the first nine years of data and is shown in Figure 2. We observe a dip or ankle at $10^{18.67 \pm 0.02} \mathrm{eV}$ and the GZK break at $10^{19.81 \pm 0.04} \mathrm{eV}$. Integrating an unbroken line from the break to $10^{21} \mathrm{eV}$, one would expect to find $\sim 80$ events in this range, while we actually observe 22 events. The significance of the cut-off is $\sim 7 \sigma$. The simplest explanation for the combination of spectral features is that of interaction between extra-galactic cosmic ray protons and the cosmic microwave background radiation (CMBR) attenuating the flux via photo-pion production above the cut-off (via a $\Delta$ resonance), and by $\mathrm{e}^{+} \mathrm{e}^{-}$pair production above the ankle [4].

The Telescope Array spectral measurements in excellent agreement with the previous measurements by the High Resolution Fly's Eye (HiRes) experiment. The Telescope Array spectrum is also largely in good agreement with the Pierre Auger measurements presented at the 2015 ICRC if one normalizes the PAO spectrum up by $16 \%$ in energy (well within the uncertainty of the experiments). In this case the two spectra overlap nicely over the ankle region and up to about $10^{19.5} \mathrm{eV}$. However, the Auger spectrum begins its cut-off significantly earlier than the Telescope Array and above this energy the two spectra diverge. Understanding this discrepancy is one area of joint study between the two groups. [?]

In an attempt to connect to the direct measurements of cosmic rays and to better understand the galactic to extra galactic transition, we have constructed the Telescope Array Low Energy Extension (TALE). One needs to look higher in the sky, since lower energy events peak at lower 


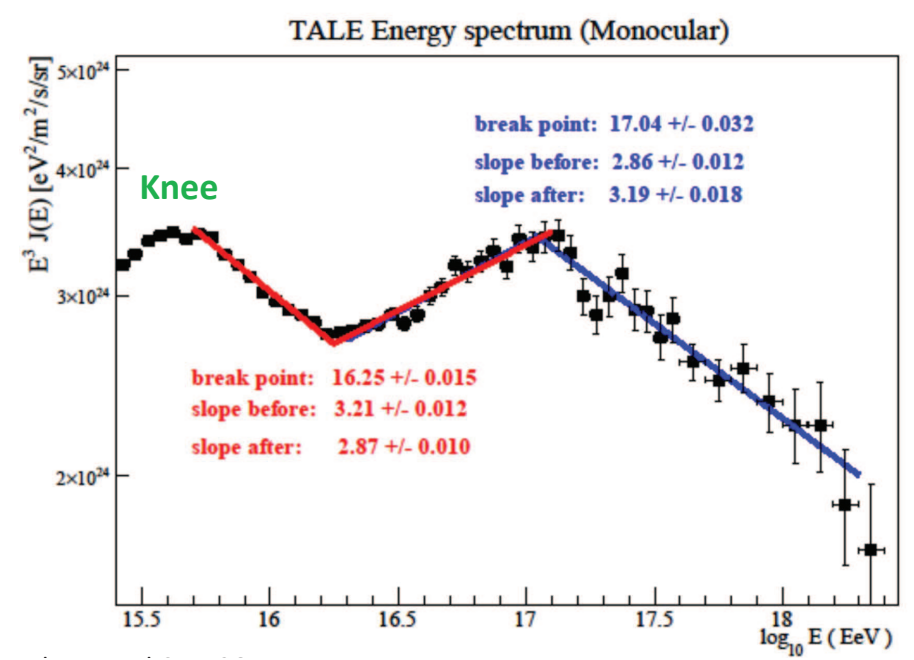

Figure 3: The Telescope Array Low Energy Extension (TALE) Energy Spectrum. The spectrum, which goes as low in energy as the Knee, shows a second knee at $10^{17.04} \mathrm{eV}$ as well as second dip $10^{16.25} \mathrm{eV}$.

grammage, higher in the sky. Therefore, at the northern (Middle Drum) telescope site, we added ten additional, high elevation angle telescopes. These view the sky above the main Telescope Array telescopes already at the site and thus above the surface detector array. The new TALE telescopes view $31-59^{\circ}$ in elevation and are re-utilized HiRes-II telescopes which have FADC electronics with 100ns sampling period. Together with the main Telescope Array telescopes, the station views 3-59 in elevation and has full azimuthal view over the SD array. These telescopes were installed in 2013 and by fall of that year they were operational.

The size of the footprint which the cosmic ray shower makes also falls as the energy of the primary cosmic ray falls. Therefore, we have added a graded array of surface detectors in front of this telescope station. The array starts with detectors spaced at $400 \mathrm{~m}$ closest to the telescope station where we expect to be looking at the lowest energy cosmic rays. The spacing grows to $600 \mathrm{~m}$, and eventually $1200 \mathrm{~m}$ where the infill array meets the main Telescope Array scintillator surface detector array. The TALE SD array is composed of the same $3 \mathrm{~m}^{2}$ scintillator detectors as the main Telescope Array SD array. An engineering/test array of 16 detectors was installed with the $400 \mathrm{~m}$ spacing at the same time as the telescopes of TALE. However, money for the rest of the 103 TALE SD detectors came later, and the full array of 103 was only deployed in early 2017 and is being commissioned in the summer of 2017. [11]

Historically, from Fly's Eye and HiRes times, we were used to discarding events with a significant amount of Čerenkov light contamination during the reconstruction of events. However, with advances of Hess, Magic, and Veritas, the Čerenkov models are much improved and we have been using events with more and more Čerenkov light. This has allowed us to push the energy threshold of our measurements much lower. With fluorescence dominated events, one can analyze events down to $\sim 10^{17.3} \mathrm{eV}$. However, using events that not only contain Čerenkov light, but are are dominated by Čerenkov light, one can reconstruct and analyze events down to nearly $10^{15} \mathrm{eV}$. This is what we have been doing with the TALE data. [12] 


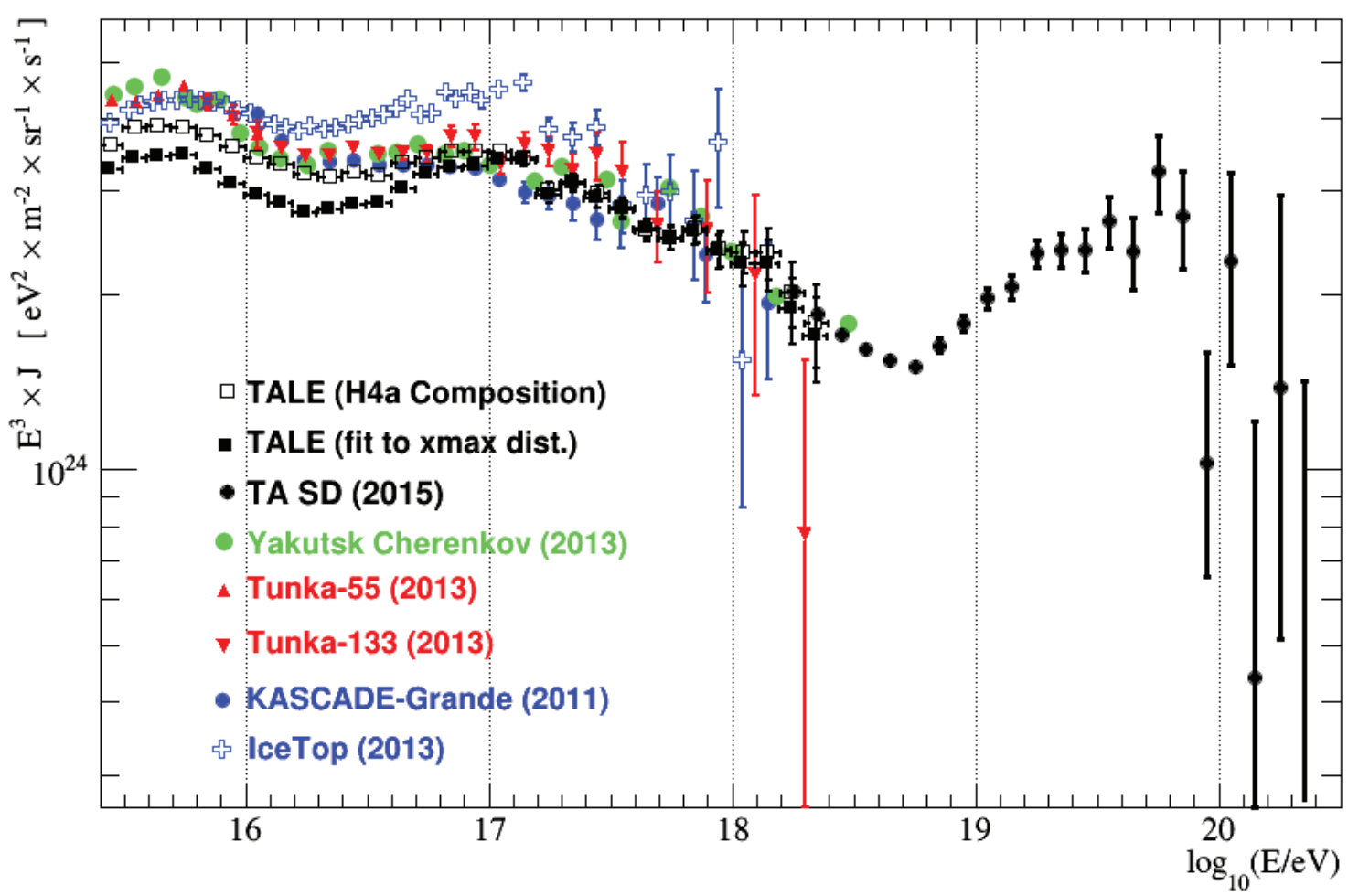

Figure 4: The Cosmic Ray Energy Spectrum from $10^{15.5} \mathrm{eV}$ to the highest energies. The full Telescope Array plus TALE energy spectrum shows The spectrum over nearly five orders of magnitude as measured by one cross-calibrated set of detectors. It shows five spectral features starting with the knee and continuing up through the GZK suppression. The TALE spectral measurements are shown for two cases: a)Using the assumption of the H4a composition and b)Using the Xmax distribution as measured by TALE to determine the composition which goes into the Monte Carlo. The TALE measurements of mean Xmax and the Ice Top measurements imply a composition which is lighter than the H4a model. In the low-energy region, measurements of the spectrum by Yakutsk, Tunka, KASKADE-Grande, and Ice Top are overlaid. The other measurements have shape similar to the TALE spectrum, however the normalizations differ.

The TALE spectrum, shown in Figure 3, demonstrates an apparent break just above $10^{17} \mathrm{eV}$ which is consistent with the "Second Knee" feature [5]. Another dip feature is seen just above the $10^{16} \mathrm{eV}$, which has been previously reported by other experiments $[6,7]$.

In Figure 4 the TALE spectrum is shown with the Telescope Array SD spectrum. The full range of the detectors covers more than five orders of magnitude in energy with one cross-calibrated set of detectors. At the low end is the cosmic ray knee and at the upper end is the GZK suppression. Yakutsk, Tunka, KASKADE-Grande, and Ice Top measurements are overlaid at the low energy end of the spectrum. They show the same general shape as the TALE spectral measurements. The absolute normalization and fine structure of the shape are still being worked on, but the data implies that the mean Xmax of air showers in this energy region is deeper in the sky (thus lighter in mass) than models such as H4a would indicate. Thus far, the geometry of these low energy events is being constrained using the distribution of Čerenkov light and a constraint on the longitudinal shape of 
the shower profile. With the newly installed TALE SD array, we will soon be able to perform true hybrid measurement of these events and have a much better measurement of the Xmax distribution and the chemical composition of these events.

Another addition coming to TALE is the NICHE detectors. These are Non-Imaging Čerenkov light detectors. A small test array of 15 detectors is currently being added on a $70 \mathrm{~m}$ grid very close to the TALE telescopes. Each detector consists of a 3 inch PMT at the bottom of a 5 inch Winston cone. They operate by solar and battery and use radio communications in the same way as the SD array. They will find air shower footprints and timing information for very low energy events which hit the ground very close to the telescope station. Construction of this test array has started and will be completed in the fall of 2017. [13]

\section{Composition}

We measure the composition using the Xmax technique. That is, we measure the depth of shower maximum in the atmosphere and we use Monte Carlo models to correlate this with chemical composition of the primary cosmic ray. Protons have a smaller cross-section and interact deeper than heavier nuclei. The Xmax distribution from proton primaries is also expected to have greater variation/width to it than, for example, iron primaries for the same reason. There is, however, considerable overlap to the Xmax distributions from the various possible components, therefore one cannot determine composition on an event by event basis. As a result, one mostly looks at mean Xmax behavior and width behavior for sets of events such as within an energy range. The mean Xmax distribution shifts deeper as a function of energy and this is called elongation.

To measure composition, one requires hybrid or stereo data in order to sufficiently constrain the geometry and thereby to make a well understood measurement of Xmax, the depth of shower maximum. In the case of a stereo measurement, the shower detector planes are intersected to constrain the geometry of the event. One advantage of stereo data is that it also provides a redundant measurement of Xmax which allows verification of the resolution measurement. With hybrid data, one extends the timing information in the angle vs. time plots which one uses to measure the pointing direction of the incoming cosmic ray. In addition, one knows the core location of the shower footprint on the Earth's surface. These additional constraints tie down the geometry.

As of 2010, the two main measurements of composition of ultra high energy cosmic rays came from the High Resolution Fly's Eye/HiRes and Pierre Auger. The High Resolution Fly's Eye/HiRes group determined that the composition was very light - consistent with protons from $10^{18.2} \mathrm{eV}$ to $10^{19.7} \mathrm{eV}$, as high in energy as they could reach. The HiRes data is, in fact, in quite good agreement with the QGSjet-01 proton model. It is also in good agreement with the QGSjetII.03 proton model. Meanwhile, the Auger group found that the composition was light in the lower energy range around $10^{18} \mathrm{eV}$. However they found that a composition which become heavier and heavier for $\mathrm{E}>\sim 3 \times 10^{18} \mathrm{eV}$ was a better interpretation of their data.

The HiRes measurements had been made using a stereo measurement from two telescope sites about $12 \mathrm{~km}$ apart. The Auger measurement had been made using hybrid measurements with telescopes and water tank surface detectors. The Telescope Array was now in a position to make hybrid measurements to test if the technique contributed to any of the difference. In order to ensure event reconstruction quality, especially with respect to Xmax determination, we instituted a 

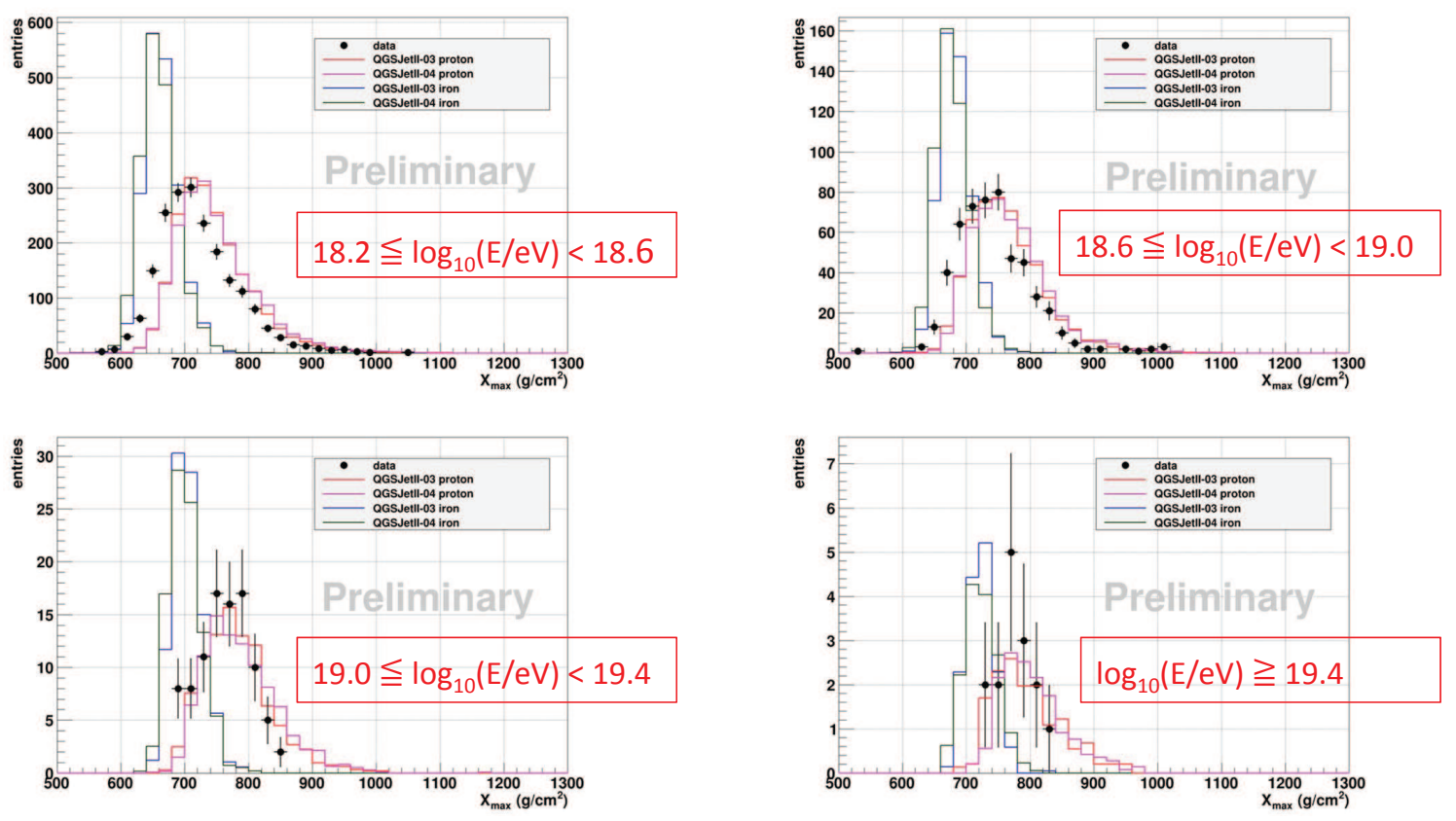

Figure 5: Data - Monte Carlo comparisons for Telescope Array Hybrid measurements of Xmax. Data (Black points) is compared to proton and iron models using the QGSjet-II.03 and QGSjetII.04 hadronic models. The comparisons are shown in four energy slices between $10^{18.2} \mathrm{eV}$ and $10^{19.4} \mathrm{eV}$. The proton model is shown as red, QGSjet-II.03 and magenta, QGSjet-II.04 histograms. The iron model is shown as blue, QGSjet-II.03 and gray, QGSjet-II.04 histograms. The data looks light and its match to protons (either model)improves with energy. We note that at the higher energies, where statistics are lacking, the data does not really fill in the tails of the distribution demonstrated by the Monte Carlo. This likely affects the composition determination.

pattern recognition cut to verify that we had a good view of both the rise and the fall of the shower development profile. With these cuts, the Xmax resolution is better than $\sim 25 \mathrm{gm} / \mathrm{cm}^{2}$ over all energies.

Figure 5 shows data-Monte Carlo comparisons of Xmax distributions for Telescope Array data measured in hybrid mode. The data is compared to both proton and iron models using the QGSjetII.03 and QGSjet-II.04 hadronic models. The comparisons are shown in four energy slices between $10^{18.2} \mathrm{eV}$ and $10^{19.4} \mathrm{eV}$. While the data is not a perfect match for the proton models, especially in the lowest energy bin, the data looks much more like protons than iron. As the energy increases, the data looks more and more like the proton models both for QGSjet-II.03 and QGSjet-II.04. At the higher energies, the quantity of data is insufficient to fully flesh out the tails of the distribution observed in the Monte Carlo.

The Xmax of each event is shown as a function of energy in Figure 6. This demonstrates the full distribution of the data. For each energy bin, the mean and width of the distribution is overlaid for the data as well as for a variety of Monte Carlo compositions. This is called the elongation. The data looks light, closer to helium than to protons. However, astrophysically helium and protons are very different. The interaction length of helium drops rapidly above $10^{19} \mathrm{eV}$, falling from about 


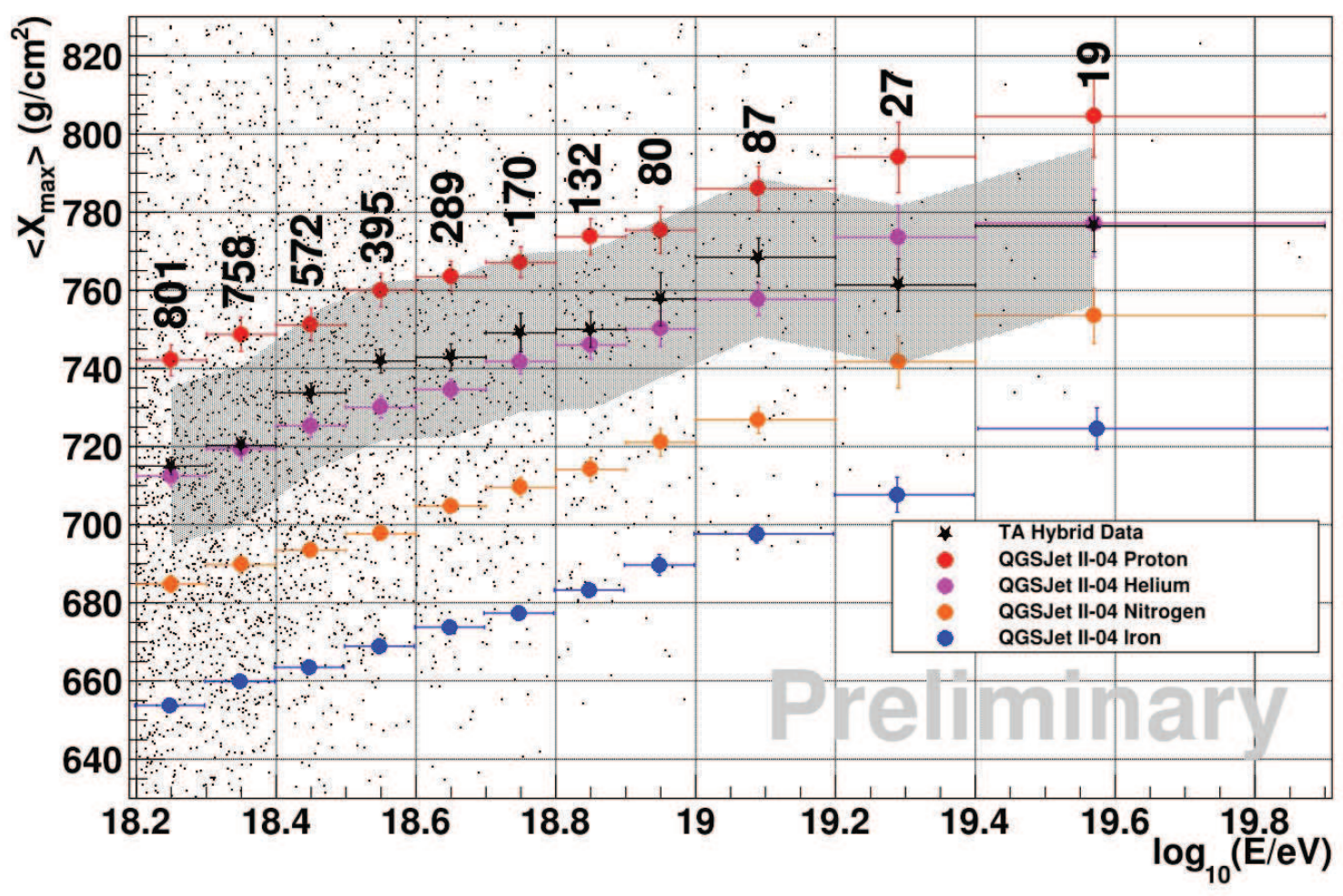

Figure 6: The Elongation of Data and Monte Carlo from the Xmax Distribution for Hybrid Measurements. The Xmax distribution of data as a function of energy is shown as the small black points. The mean and uncertainty of the data distribution in energy bins is shown by black stars with error bars. The mean and uncertainty of the simulations using QGSjet-II.04 hadronic generator are shown for protons (red), helium (magenta), nitrogen (orange), and iron (blue). The data largely lies between proton and helium simulations. Above $10^{19} \mathrm{eV}$ the uncertainty grows substantially due to low statistics.

$1000 \mathrm{Mpc}$ to about a Mpc. Hydrogen, on the other hand, maintains a high interaction length to nearly $10^{20} \mathrm{eV}$. When the hydrogen interaction length does fall, dropping to $10 \mathrm{~s} \mathrm{of} \mathrm{Mpc,} \mathrm{it} \mathrm{is} \mathrm{still}$ much longer than the helium.

Figure 7 shows the width of the Xmax distribution in energy steps for the same data. It compares those widths to the expected widths for QGSjet-II.04 Monte Carlo models of protons, helium, nitrogen, and iron. The data looks very light/protonic. At the highest energies, the uncertainties again become larger due to poor statistics. See W.Hanlon presentation for more information. [16]

The two hybrid and the stereo measurements of Xmax are in good agreement with one another as well as indicating a predominately light composition. The interpretation of a light composition is fairly robust, as illustrated by the scales shown in Figure 8. To leading order, the departure of the data points from the proton line is roughly proportional to $\ln (\mathrm{A})$ where $\mathrm{A}$ is the nuclear mass number. Even if the scale were set to a hypothetical model that has a proton rail that is $33 \%$ deeper, the data points shown in the plot would only reach that expected for helium in this scenario. However, protons and helium are very different astrophysically as previously pointed out. 


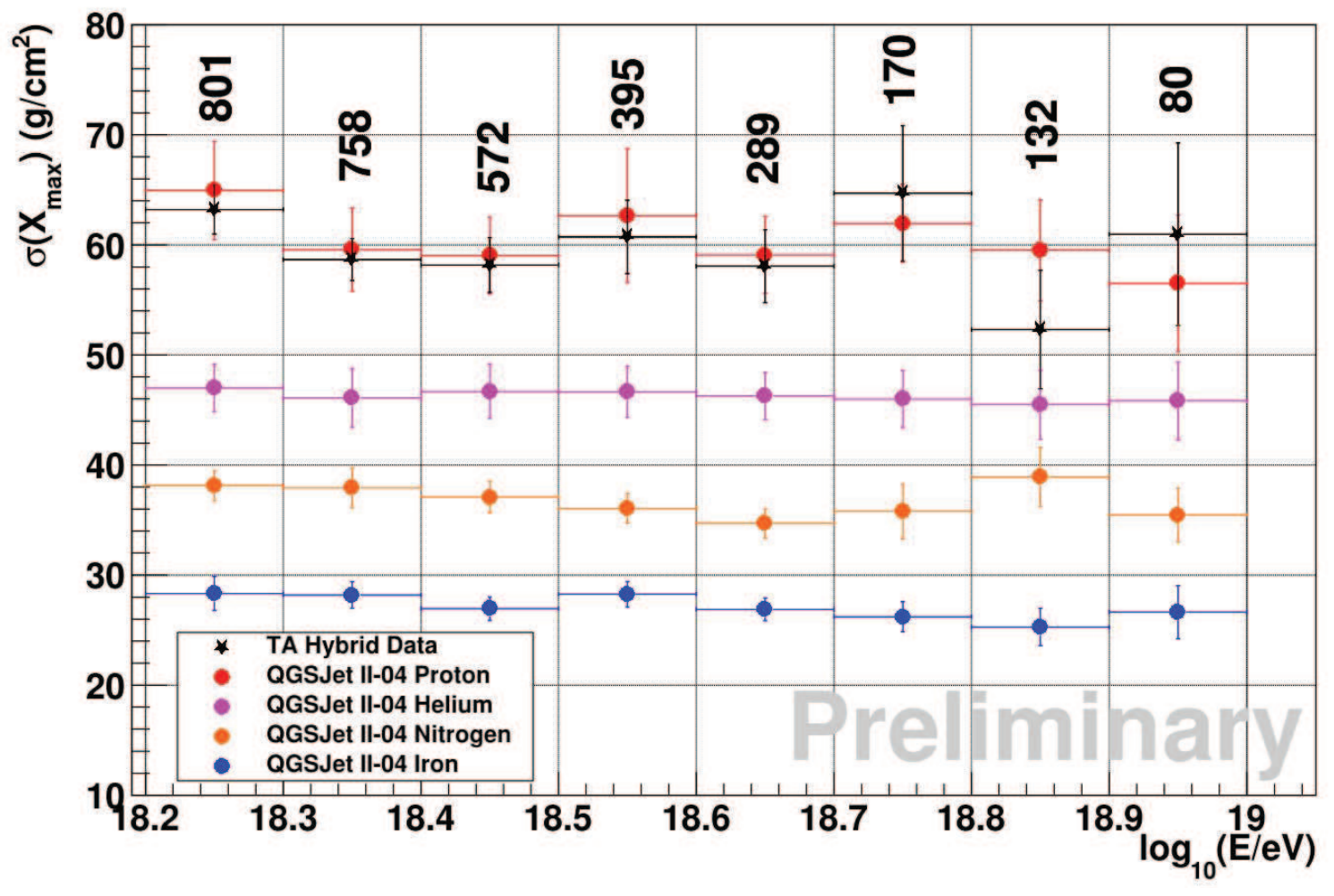

Figure 7: The Data - Monte Carlo comparison of the width of the Xmax Distribution for Hybrid Measurements. The width of the data distributions is compared to the expected widths for QGSjetII.04 Monte Carlo models of protons (red), helium (magenta), nitrogen (orange), and iron (blue). The data looks very light/protonic. At the highest energies, the uncertainties again become large due to poor statistics.

\section{Anisotropy}

The Telescope Array collaboration has previously published that we have observed indications of a potential hot spot in the arrival direction of ultra high energy cosmic rays. [9] This publication used the data from the first five years of SD operations. Using the Auger criteria for their published AGN correlations, we observed 72 events with $\mathrm{E}>5.7 \times 10^{19} \mathrm{eV}$. These events are then oversampled using $20^{\circ}$ circles. We find that 19 of the 72 events overlap a region, we call the hot spot, which is centered at $146.7^{\circ}$ in right ascension and $43.2^{\circ}$ declination. Thus, $26 \%$ of events are in about $6 \%$ of the observable area. We expect a background of 4.5 isotropic events in this area. The Li-Ma significance of this is $\sim 5.2 \sigma$. Taking into account that this spot could appear anywhere in the sky gives us a chance probability estimate of $3.4 \sigma$.

The hot spot is about $19^{\circ}$ off of the super-galactic plane, near Ursa Major. The Ursa Major super-cluster is extended more than $\pm 10^{\circ}$ from the super-galactic plane. Therefore, we can not rule out some sort of relationship between the hot spot and this super-cluster.

One interesting thing to do is to make a full-sky map combining the Telescope Array and Pierre Auger events with E $>5.7 \times 10^{19} \mathrm{eV}$. See Figure 9. The plot is shown in Hammer-Aitoff projection, in Equatorial coordinates. No correction was made for the energy scale difference 


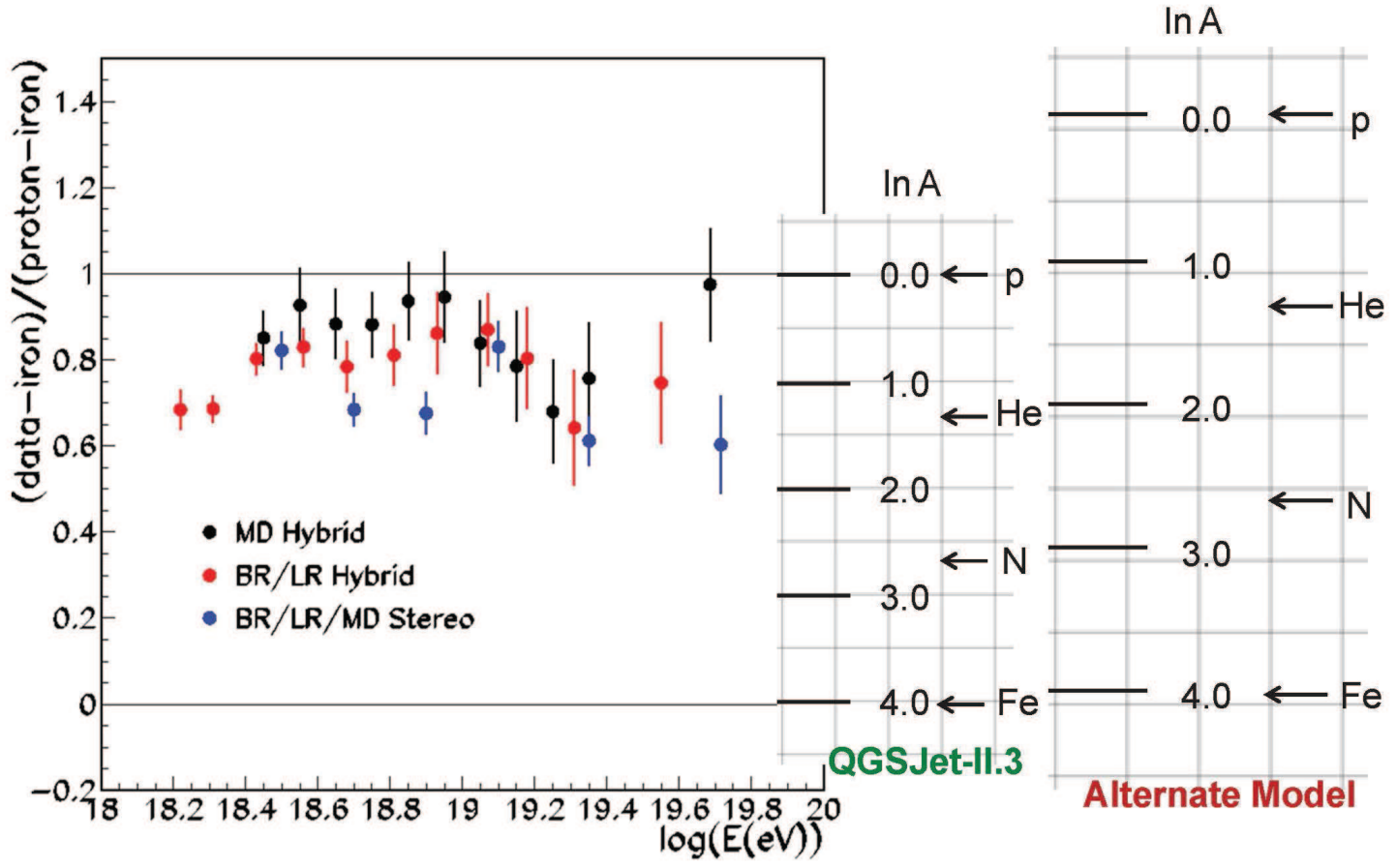

Figure 8: Summary of average $X_{M A X}$ from Telescope Array fractional as the fractional position between the expectations of pure iron (lower lines at zero) and pure proton (upper line at 1.0) using the QGSjet-II.03 hadronic model. At right is the illustrative interpretation, in terms of $\ln A$ of the summary plot against QGSjet-II.03 and an alternative model where the proton rail is about 33\% deeper in $X_{M A X}$.

between the Telescope Array and Pierre Auger data sets. The thin gray line just above and left of the Telescope Array (upper) hotspot is the supergalactic plane. The pre-trial significance of this is $5.2 \sigma$ (post-trial chance probability of $\sim 3.4 \sigma$ ). The Auger "warm spot" (lower) is located on the supergalactic plane at Centaurus-A and has a pretrial significance of $\sim 3.6 \sigma$. Neither the Telescope Array, nor Pierre Auger data shows any sign of excess in the direction of Virgo.

We now update the measurement by adding the four additional years of data, for a total of nine years, collected through spring 2017. The search was first performed, just as before, with the original $20^{\circ}$ oversampling, however it was apparent that the hot spot looked larger. See Figure ??. Since the hot spot was something that we noticed in the data and there was no a priori source there, we decided to scan over the oversampling size. When we wrote the original paper [9], we had scanned over oversampling size for the Monte Carlo, but had not scanned over the data. Now we scanned over the data. In both cases, the scanning was limited to $15^{\circ}, 20^{\circ}, 25^{\circ}, 30^{\circ}$, and $35^{\circ}$ oversampling. The maximum significance came with $25^{\circ}$ oversampling.

There are now a total of 143 events with $\mathrm{E}>5.7 \times 10^{19} \mathrm{eV}$. Of these, 34 events are within the hot spot, while we have an expected background of 13.5 events. The local significance is $5 \sigma$ and global significance is $3 \sigma$, not much different than was found previously for the first 5 years. A Kolmagorov-Smirnov test shows that the rate of arrival of events to the hot spot is consistent with the fluctuations expected from a Poisson distribution with a mean of 2.8 events/year. The rate 


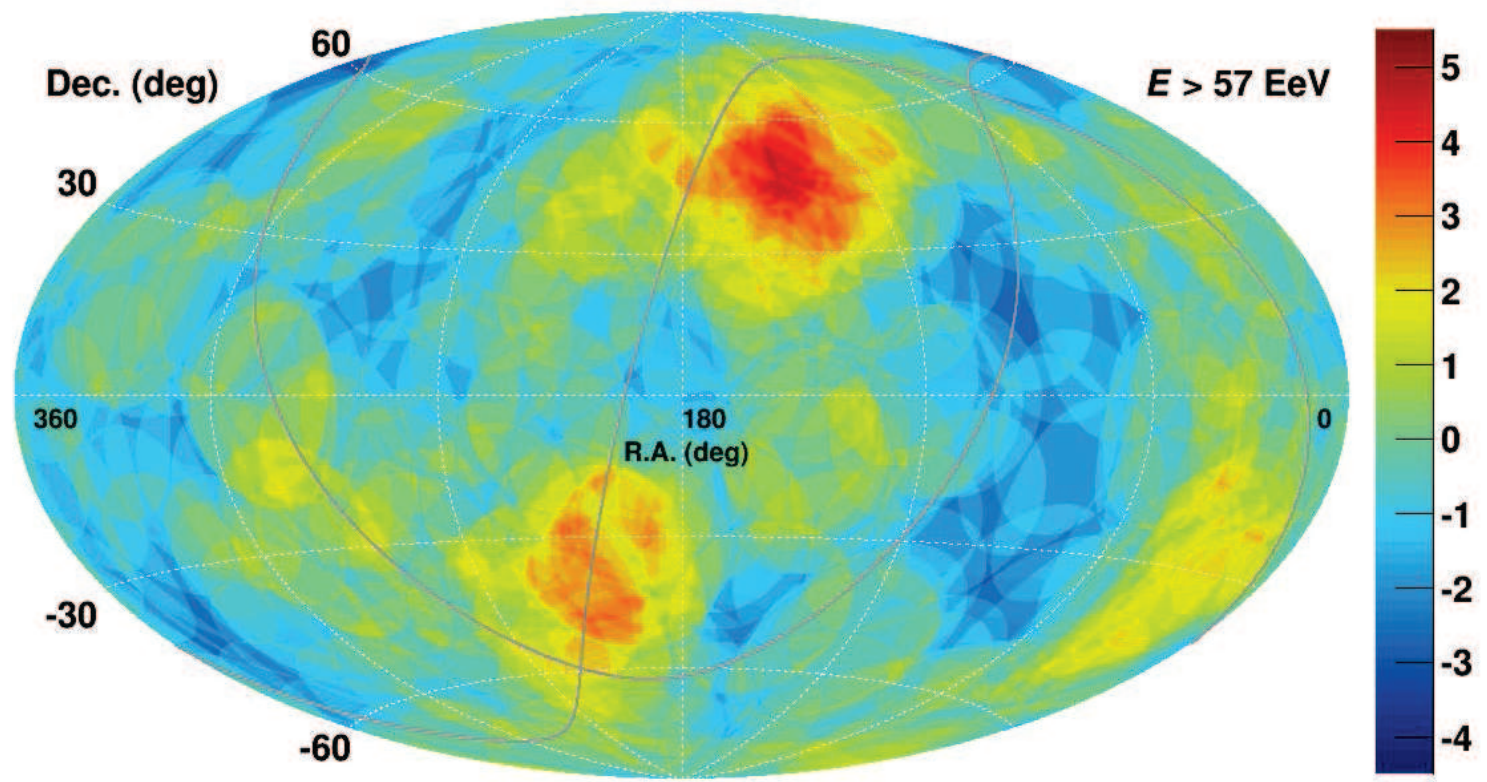

Figure 9: Full sky map combining the Telescope Array and Pierre Auger data events with E $>5.7 \times 10^{19} \mathrm{eV}$. The events have oversampling with a $20^{\circ}$ radius circle. The Telescope Array data set includes 109 events, representing the first 7 years of data collection. The Auger data set includes 157 events, representing 10 years of data. No correction was made for the energy scale difference between the Telescope Array and Pierre Auger data sets. The thin gray line just above and left of the Telescope Array (upper) hot spot is the supergalactic plane.

of event arrivals is also inconsistent with chance excess from an isotropic distribution, a Poisson average of 0.9 events/year, at about $2.6 \sigma$. It is clear that we need a lot more data to sort this out.

We have also looked for correlation with the Large Scale Structure (LSS). This is done by comparing to the matter distribution observed by the 2MASS Galaxy redshift catalog (XSCz). The sky was divided into five intensity regions, these were then compared by a KolmagorovSmirnov test comparing to the expected flux distribution. For events with energies, $\mathrm{E}>10^{18} \mathrm{eV}$ and $\mathrm{E}>4 \times 10^{19} \mathrm{eV}$, the test can not distinguish between the Large Scale Structure and isotropy. However, for events $\mathrm{E}>5.7 \times 10^{19} \mathrm{eV}$, the KS test indicated that the data is marginally incompatible with an isotropic source distribution and it is compatible with the LSS simulation. See S.Troitsky and D.Ivanov presentations for more information. [14, 15]

\section{Future Plans}

The collaboration has plans to quadruple the size of the Telescope Array. We are adding 500 scintillator surface detectors to the North East and South East of the present Telescope Array site. See Figure 11. The detectors will be on a grid a $2.08 \mathrm{~km}$ spacing and so will focus on the highest energy region. This part of the expansion has been funded in 2015 by the Japanese funding agencies. There will also be two new telescope stations which will view the sky over this expansion. This was also funded in 2016 by the US NSF. The first 100 scintillator detectors already arrived in Delta, Utah and 70 more are currently being assembled in the Akeno Observatory of the University 


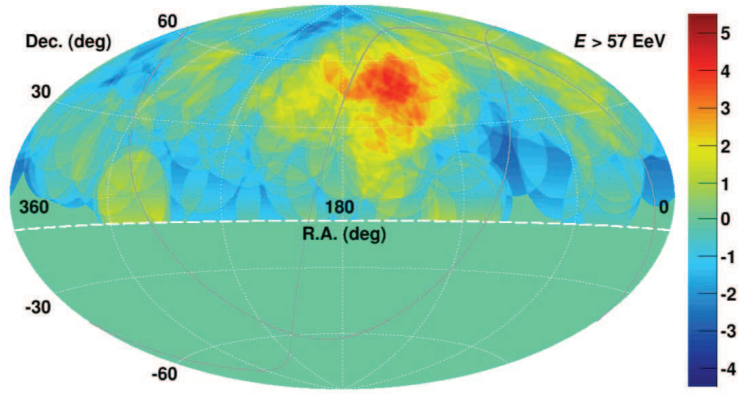

With original $20^{\circ}$ oversampling, spot looks larger.... Thus, scan over $15^{\circ}, 20^{\circ}, 25^{\circ}, 30^{\circ}$, $\& 35^{\circ}$

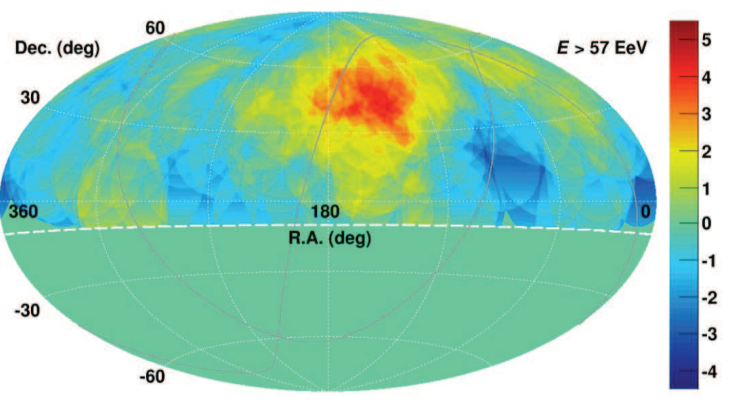

With $25^{\circ}$ oversampling, significance maximum $3 \sigma$

\begin{tabular}{|l|l|l|l|l|l|l|l|l|l|l|}
\hline Binsize & $\mathbf{1 5}$ & $\mathbf{2 0}$ & $\mathbf{2 5}$ & $\mathbf{3 0}$ & $\mathbf{3 5}$ \\
\hline & Local & Global & Local & Global & Local & Global & Local & Global & Local & Global \\
\hline Year 5 & 5.12 & 3.14 & 5.43 & 3.55 & 5.16 & 3.19 & 4.82 & 2.73 & 4.33 & 2.05 \\
\hline Year 7 & 4.92 & 2.84 & 5.37 & 3.44 & 5.65 & 3.80 & 5.37 & 3.44 & 5.03 & 2.99 \\
\hline Year 9 & 4.42 & 2.06 & 4.72 & 2.50 & 5.06 & 2.96 & 5.01 & 2.91 & 4.66 & 2.41 \\
\hline
\end{tabular}

Figure 10: Left: The sky map of Telescope Array events with $\mathrm{E}>5.7 \times 10^{19} \mathrm{eV}$ from the first nine years of data. The data has an oversampling of $20^{\circ}$ as in the original analysis. The hot spot is noticeably larger than in the first 5 years of data. Right: The sky map of Telescope Array events with $\mathrm{E}>5.7 \times 10^{19} \mathrm{eV}$ from the first nine years of data. The data has an oversampling of $25^{\circ}$ which showed the maximum significance, about $5 \sigma$ local, $3 \sigma$ global.

of Tokyo and will be shipped to Utah in the fall of 2017. We are working with the US Bureau of Land Management to get the lease to the sites. With this enlarged Telescope Array detector, we will be able collect the equivalent of 19 current Telescope Array SD years of data by 2020. With the new data from the TA $\times 4$, we will be able to quickly verify the hotspot and to begin searching for sub-structure within the hotspot. If there are really two separated sources there, we would begin to be able to resolve this.

\section{Summary}

The Telescope Array has measured the energy spectrum, composition, and arrival direction of ultra high energy cosmic rays in the northern hemisphere. The low energy extension, TALE is coming on line and we are pushing the energy threshold of the experiment down below $10^{16} \mathrm{eV}$. The Telescope Array and TALE have already measured the energy spectrum between $6 \times 10^{15} \mathrm{eV}$ to more than $10^{20} \mathrm{eV}$ with a single set of cross calibrated detectors. By looking at the composition and the energy spectrum in this range, we hope to identify the galactic to extragalactic transition. The surface array portion of TALE was recently deployed and this will give us the capability to do hybrid composition in this energy range. The composition of cosmic rays is consistent with a light composition for $\mathrm{E}>10^{18.2} \mathrm{eV}$ from both hybrid and stereo measurements. We have also reported on a hotspot near the direction of Ursa Major with a $\sim 3 \sigma$ global significance. It now appears larger 
than we originally thought. We need more data to verify the source and to better to resolve it. More data is needed, but more data is coming with the expansion of TA $\times 4$.

The Telescope Array experiment is supported by the Japan Society for the Promotion of Science through Grants-in-Aids for Scientific Research on Specially Promoted Research (21000002) "Extreme Phenomena in the Universe Explored by Highest Energy Cosmic Rays" and for Scientific Research (19104006), and the Inter-University Research Program of the Institute for Cosmic Ray Research; by the U.S. National Science Foundation; by the National Research Foundation of Korea (2007-0093860, R32-10130, 2012R1A1A2008381, 2013004883); by the Russian Academy of Sciences, RFBR grants 11-02-01528a and 13-02-01311a (INR), IISN project No. 4.4502.13 and Belgian Science Policy under IUAP VII/37 (ULB). The foundations of Dr. Ezekiel R. and Edna Wattis Dumke, Willard L. Eccles and the George S. and Dolores Dore Eccles all helped with generous donations. The State of Utah supported the project through its Economic Development Board, and the University of Utah through the Office of the Vice President for Research. The experimental site became available through the cooperation of the Utah School and Institutional Trust Lands Administration (SITLA), U.S. Bureau of Land Management, and the U.S. Air Force. We also wish to thank the people and the officials of Millard County, Utah for their steadfast and warm support. We gratefully acknowledge the contributions from the technical staffs of our home institutions. An allocation of computer time from the Center for High Performance Computing at the University of Utah is gratefully acknowledged.

\section{References}

[1] T. Abu-Zayyad et al. (Telescope Array Collaboration), Astrophys. Journal Letters 768, L1 (2013).

[2] K. Greisen, Phys. Rev. Lett. 16, 222 (1966).

[3] G.T. Zatsepin and V.A. Kuz'min, JETPL 4, 78 (1966).

[4] V. Berezinsky, A.Z. Gazizov, and S.I. Grigorieva, Physics Letters B 612, 147 (2005).

[5] D.R. Bergman and J.W. Belz, J.Phys. G34, R359 (2007).

[6] W.D. Apel et al. (KASCADE-Grande Collaboration) Astropart. Phys. 36, 183 (2012).

[7] M.G. Aartsen et al. (IceCube Collaboration) Phys. Rev. D 88, 042004 (2013).

[8] R.U. Abbasi et al. (Telescope Array Collaboration), Astropart. Phys. 64, 49 (2015).

[9] R.U. Abbasi et al. (Telescope AArray Collaboration), Astrophysical Journal Lett. 790, L21 (2014).

[10] T. Abu-Zayyad et al. (Telescope Array Collaboration), Astrophysical Journal 777, 88 (2013).

[11] S. Udo et al. (for the Telescope Array Collaboration), Proceedings of the 2017 ICRC, Busan, South Korea, CRI-069.

[12] T.AbuZayyad et al. (for the Telescope Array Collaboration), Proceedings of the 2017 ICRC, Busan, South Korea, CRI-126.

[13] Y. Tsunesada et al. (for the Telescope Array Collaboration), Proceedings of the 2017 ICRC, Busan, South Korea, CRI-110.

[14] S. Troitsky et al. (for the Telescope Array Collaboration), Proceedings of the 2017 ICRC, Busan, South Korea, CRI-047. 
[15] D.Ivanov et al. (for the Telescope Aarray Collaboration), Proceedings of the 2017 ICRC, Busan, South Korea, CRI-236.

[16] W,Hanlon et al. (for the Telescope Array Collaboration), Proceedings of the 2017 ICRC, Busan, South Korea, CRI-123.

[17] D.Ivanov et al. (for the Pierre Auger and Telescope Array Collaborations), Proceedings of the 2017 ICRC, Busan, South Korea, CRI-231. 


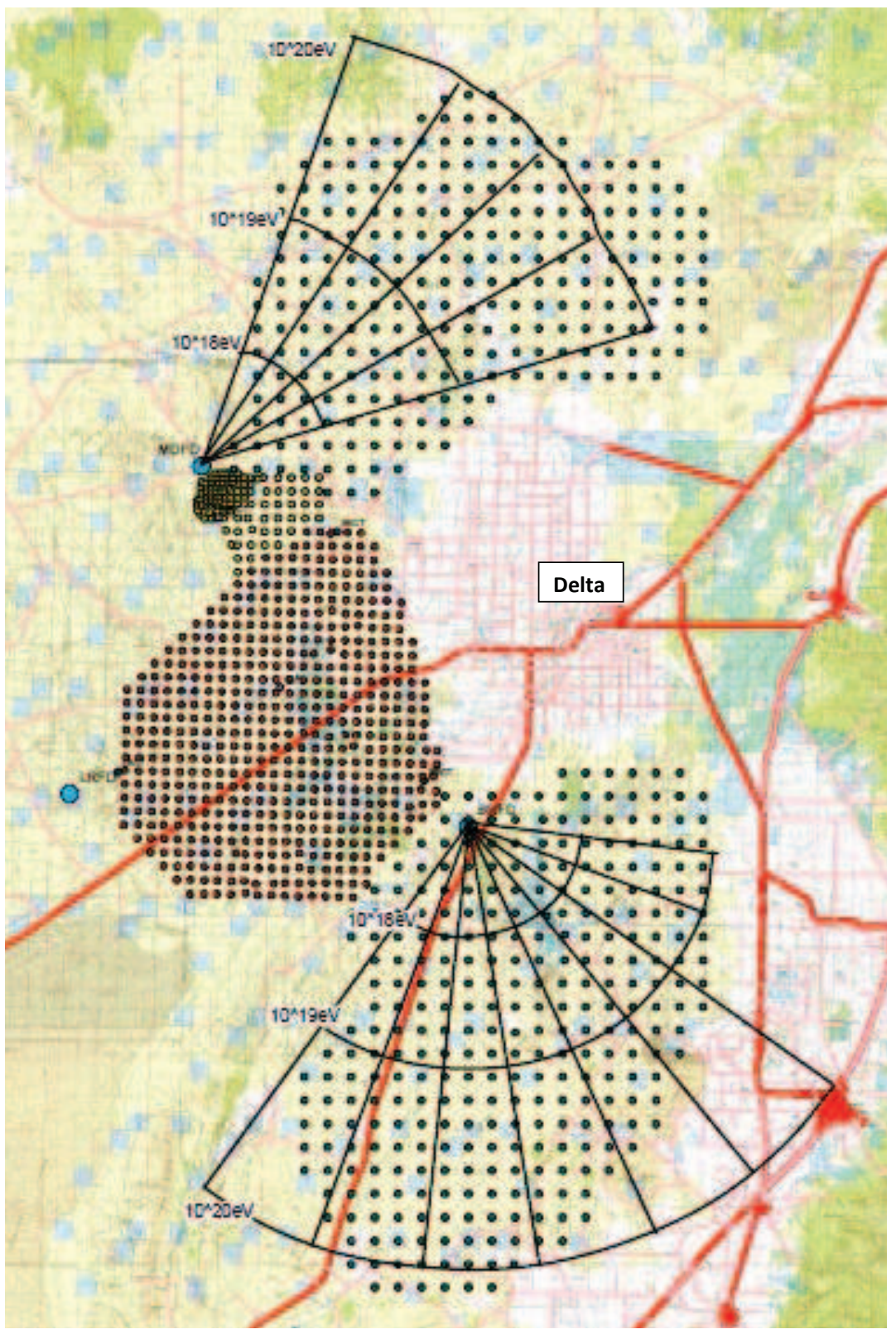

Figure 11: Map showing the Telescope Array with the high energy expansion, TA $\times 4$. The main Telescope Array with $1.2 \mathrm{~km}$ spacing is shown with the red points. The TALE, Telescope Array Low Energy Extension is shown as yellow points. This is a graded array with 1.2, 0.6 and 0.4 $\mathrm{km}$ spacing. The TA $\times 4$ expansion is shown to the NE and SE of the main Telescope Array with the dark points at $2.08 \mathrm{~km}$ spacing. The large blue points indicate the telescope stations. New telescopes will be added to the northern (Middle Drum) and south-eastern (Black Rock Mesa) telescope sites to view the sky over the TA $\times 4$ scintillator array expansion. The field of view of the 12 new telescopes is shown as the black fan with radii drawn to indicate the rough viewing distance for a vertical shower with energy $10^{18}, 10^{19}$, and $10^{20} \mathrm{eV}$. The white space (with many red lines roads) to the right of the main Telescope Array is the town of Delta. 